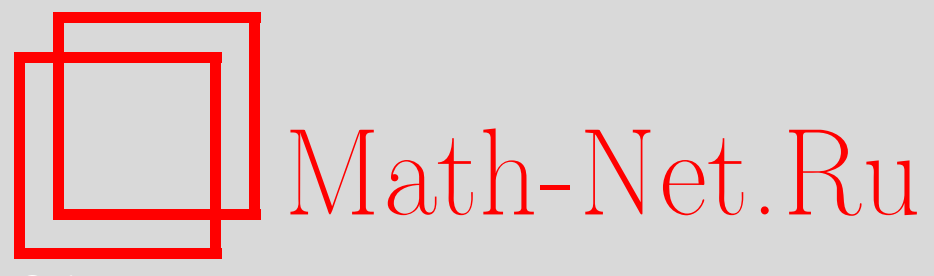

Л. Н. Орлова, Геометрия квазилинейной системы двух дифференциальных уравнений с частными производными первого и второго порядков при двух функциях от двух независимых переменных, Матем. заметки, 2009, том 85, выпуск 3, 421-432

DOI: https://doi.org/10.4213/mzm3914

Использование Общероссийского математического портала Math-Net.Ru подразумевает, что вы прочитали и согласны с пользовательским соглашением http: //www.mathnet.ru/rus/agreement

Параметры загрузки:

IP : 54.162 .27 .143

26 апреля 2023 г., 12:45:47

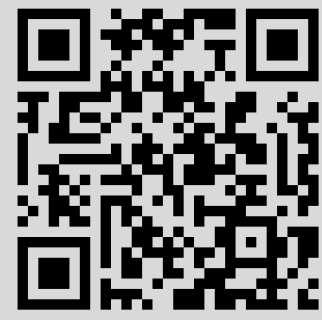


Том 85 выпуск 3 март 2009

УДК 517.958

\section{Геометрия квазилинейной системы двух дифференциальных уравнений с частными производными первого и второго порядков при двух функциях от двух независимых переменных}

\section{Л. Н. Орлова}

В работе изучается геометрия системы двух дифференциальных уравнений с частными производными первого и второго порядков при двух функциях от двух независимых переменных, при этом используется метод инвариантных форм Эли Картана, а также теоретико-групповой метод “продолжений и охватов" Г. Ф. Лаптева (для конечных групп) и А. М. Васильева (для бесконечных групп). Приведена классификация систем квазилинейных уравнений первого и второго порядков при двух функциях $u$ и $v$ от двух независимых переменных $x$ и $y$.

Библиография: 10 названий.

Условие задачи. Пусть дана система двух дифференциальных уравнений с частными производными при двух функциях $(u, v)$ от двух независимых переменных $(x, y)$. Одно уравнение - первого порядка, другое - второго порядка. Уравнения заданы в общем виде:

$$
\begin{aligned}
F\left(x, y, u, v, u_{x}, u_{y}, v_{x}, v_{y}\right) & =0, \\
\Phi\left(x, y, u, v, u_{x}, u_{y}, v_{x}, v_{y}, u_{x x}, u_{x y}, u_{y y}, v_{x x}, v_{x y}, v_{y y}\right) & =0 .
\end{aligned}
$$

Изучается геометрия этой системы дифференциальных уравнений, т.е. согласно общей схеме Клейна свойства совокупности интегральных многообразий этой системы, инвариантные относительно преобразований бесконечной группы точечных преобразований

$$
\begin{aligned}
& x^{\prime}=f_{1}(x, y, u, v), \\
& y^{\prime}=f_{2}(x, y, u, v), \\
& u^{\prime}=f_{3}(x, y, u, v), \\
& v^{\prime}=f_{4}(x, y, u, v)
\end{aligned}
$$

и проводится классификация этих систем.

Изучение ведется при помощи метода внешних форм Картана [1]-[3] и теории погруженных многообразий Лаптева [4] - для конечных групп и Васильева [5], [6] для бесконечных.

Для изучения геометрии данных дифференциальных уравнений нам необходимо найти представление соответствующей бесконечной группы, которое показывает, как эта группа преобразует все входящие в уравнения переменные. А именно, 
группа (2) преобразует переменные, входящие в уравнения (1) по формулам (2), дополненным уравнениями

$$
\begin{aligned}
u_{x}^{\prime}= & \frac{\left(\frac{\partial f_{3}}{\partial x}+\frac{\partial f_{3}}{\partial u} u_{x}+\frac{\partial f_{3}}{\partial v} v_{x}\right)\left(\frac{\partial f_{2}}{\partial y}+\frac{\partial f_{2}}{\partial u} u_{y}+\frac{\partial f_{2}}{\partial v} v_{y}\right)}{A} \\
& -\frac{\left(\frac{\partial f_{3}}{\partial y}+\frac{\partial f_{3}}{\partial u} u_{y}+\frac{\partial f_{3}}{\partial v} v_{y}\right)\left(\frac{\partial f_{2}}{\partial x}+\frac{\partial f_{2}}{\partial u} u_{x}+\frac{\partial f_{2}}{\partial v} v_{x}\right)}{A}, \\
u_{y}^{\prime}= & \frac{\left(\frac{\partial f_{1}}{\partial x}+\frac{\partial f_{1}}{\partial u} u_{x}+\frac{\partial f_{1}}{\partial v} v_{x}\right)\left(\frac{\partial f_{3}}{\partial y}+\frac{\partial f_{3}}{\partial u} u_{y}+\frac{\partial f_{3}}{\partial v} v_{y}\right)}{A} \\
& \left.-\frac{\partial f_{1}}{\partial y}+\frac{\partial f_{1}}{\partial u} u_{y}+\frac{\partial f_{1}}{\partial v} v_{y}\right)\left(\frac{\partial f_{3}}{\partial x}+\frac{\partial f_{3}}{\partial u} u_{x}+\frac{\partial f_{3}}{\partial v} v_{x}\right) \\
A= & \left.\frac{\partial f_{1}}{\partial x}+\frac{\partial f_{1}}{\partial u} u_{x}+\frac{\partial f_{1}}{\partial v} v_{x}\right)\left(\frac{\partial f_{2}}{\partial y}+\frac{\partial f_{2}}{\partial u} u_{y}+\frac{\partial f_{2}}{\partial v} v_{y}\right) \\
& -\left(\frac{\partial f_{1}}{\partial y}+\frac{\partial f_{1}}{\partial u} u_{y}+\frac{\partial f_{1}}{\partial v} v_{y}\right)\left(\frac{\partial f_{2}}{\partial x}+\frac{\partial f_{2}}{\partial u} u_{x}+\frac{\partial f_{2}}{\partial v} v_{x}\right) .
\end{aligned}
$$

Аналогичные формулы получаются для $v_{x^{\prime}}^{\prime}$ и $v_{y^{\prime}}^{\prime}$. Можно также подсчитать, как выражаются $u_{x^{\prime} x^{\prime}}^{\prime}, u_{x^{\prime} y^{\prime}}^{\prime}, u_{y^{\prime} y^{\prime}}^{\prime}, v_{x^{\prime} x^{\prime}}^{\prime}, v_{x^{\prime} y^{\prime}}^{\prime}, v_{y^{\prime} y^{\prime}}^{\prime}$ через переменные $x, y, u, v, u_{x}, u_{y}$, $v_{x}, v_{y}, u_{x x}, u_{x y}, u_{y y}, v_{x x}, v_{x y}, v_{y y}$. Будем считать производные $u_{x}, u_{y}, v_{x}, v_{y}, u_{x x}, u_{x y}$, $u_{y y}, v_{x x}, v_{x y}, v_{y y}$ новыми переменными, преобразующимися по формулам (3). Тогда уравнения задачи будут содержать 14 переменных: $u_{1}=x, u_{2}=y, u_{3}=u, u_{4}=v$, $p_{1}=u_{x}, p_{2}=u_{y}, p_{3}=v_{x}, p_{4}=v_{y}, q_{1}=u_{x x}, q_{2}=u_{x y}, q_{3}=u_{y y}, q_{4}=v_{x x}, q_{5}=v_{x y}$, $q_{6}=v_{y y}$. Задание этой системы уравнений сводится к заданию подмногообразия в этом пространстве представления. Если рассматривать только переменные, входящие в первое уравнение: $u_{1}, u_{2}, u_{3}, u_{4}, p_{1}, p_{2}, p_{3}$ и $p_{4}$, то одно первое уравнение определит семимерную поверхность $M^{(7)}$ в этом восьмимерном пространстве $M^{(8)}$.

Два уравнения задачи на 14 переменных налагают два условия. Остается 12 свободных переменных. Получим многообразие $M^{(12)}$ в $M^{(14)}$. Будем считать его достаточно гладким. Для того чтобы воспользоваться методом внешних форм, нужно это многообразие задать уравнениями Пфаффа между независимыми инвариантными линейными формами соответствующей бесконечной группы. От переменных $u_{1}, u_{2}, \ldots, q_{6}$ перейдем к их дифференциалам $d u_{1}, d u_{2}, \ldots, d q_{6}$, а затем, сделав замену базиса, введем новые линейно независимые формы в том же количестве. Вместо дифференциалов $d u_{1}, d u_{2}, d u_{3}, d u_{4}$ введем формы $\omega^{1}, \omega^{2}, \omega^{3}, \omega^{4}$; вместо дифференциалов $d p_{1}, d p_{2}, d p_{3}, d p_{4}$ - базисные формы $\omega_{1}^{3}, \omega_{2}^{3}, \omega_{1}^{4}, \omega_{2}^{4}$; вместо дифференциалов $d q_{1}, d q_{2}, d q_{3}, d q_{4}, d q_{5}, d q_{6}$ - формы $\omega_{11}^{3}, \omega_{12}^{3}, \omega_{22}^{3}, \omega_{11}^{4}, \omega_{12}^{4}, \omega_{22}^{4}$. Эти новые инвариантные формы группы удовлетворяют некоторым соотношениям - уравнениям структуры Ли-Картана на внешние дифференциалы этих форм:

$$
\begin{array}{rlr}
D \omega^{i} & =\left[\omega_{k}^{i} \omega^{k}\right], \\
D \omega_{k}^{i} & =\left[\omega_{l}^{i} \omega_{k}^{l}\right]+\left[\omega_{k l}^{i} \omega^{l}\right], \quad\left[\omega_{k l}^{i} \omega^{k} \omega^{l}\right]=0, \\
D \omega_{k l}^{i} & =\left[\omega_{p l}^{i} \omega_{k}^{p}\right]+\left[\omega_{k p}^{i} \omega_{l}^{p}\right]-\left[\omega_{k l}^{p} \omega_{p}^{i}\right]+\left[\omega_{k l p}^{i} \omega^{p}\right], \quad\left[\omega_{k l p}^{i} \omega^{k} \omega^{l} \omega^{p}\right]=0, \\
& i, k, l, p=1,2,3,4 .
\end{array}
$$


Запишем дифференциалы основных уравнений задачи

$$
\begin{array}{r}
d F\left(u_{1}, u_{2}, u_{3}, u_{4}, p_{1}, p_{2}, p_{3}, p_{4}\right)=0, \\
d \Phi\left(u_{1}, u_{2}, u_{3}, u_{4}, p_{1}, p_{2}, p_{3}, p_{4}, q_{1}, q_{2}, q_{3}, q_{4}, q_{5}, q_{6}\right)=0,
\end{array}
$$

или

$$
\begin{aligned}
\frac{\partial F}{\partial u_{1}} d u_{1} & +\frac{\partial F}{\partial u_{2}} d u_{2}+\frac{\partial F}{\partial u_{3}} d u_{3}+\frac{\partial F}{\partial u_{4}} d u_{4} \\
+ & \frac{\partial F}{\partial p_{1}} d p_{1}+\frac{\partial F}{\partial p_{2}} d p_{2}+\frac{\partial F}{\partial p_{3}} d p_{3}+\frac{\partial F}{\partial p_{4}} d p_{4}=0 \\
\frac{\partial \Phi}{\partial u_{1}} d u_{1}+\cdots+ & +\frac{\partial \Phi}{\partial p_{1}} d p_{1}+\cdots+\frac{\partial \Phi}{\partial q_{1}} d q_{1}+\cdots+\frac{\partial \Phi}{\partial q_{6}} d q_{6}=0
\end{aligned}
$$

Перейдем от дифференциалов $d p_{1}, d p_{2}, d p_{3}, d p_{4}, d q_{1}, d q_{2}, d q_{3}, d q_{4}, d q_{5}, d q_{6}$ к независимым линейным инвариантным формам нашей группы:

$$
\begin{aligned}
\widetilde{A} \omega_{1}^{3}+\widetilde{B} \omega_{2}^{3}+\widetilde{C} \omega_{1}^{4}+\widetilde{D} \omega_{2}^{4}+\widetilde{K}_{i} \omega^{i} & =0, \quad i=1,2,3,4 \\
\bar{A} \omega_{11}^{3}+\bar{B} \omega_{22}^{3}+\bar{C} \omega_{11}^{4}+\bar{D} \omega_{22}^{4}+\alpha \omega_{1}^{3}+\beta \omega_{2}^{3}+\gamma \omega_{2}^{4}+e_{i} \omega^{i} & =0,
\end{aligned}
$$

Всякий так называемый геометрический обгект, инвариантно связанный с уравнением, является точкой пространства представления данной группы преобразований. Общая теория утверждает, что все они получаются внешним дифберениированием, продолжением и канонизачией этих уравнений. Если $\widetilde{C} \neq 0$, то первое из уравнений (6) можно разрешить относительно $\omega_{1}^{4}$ :

$$
\omega_{1}^{4}=A \omega_{1}^{3}+B \omega_{2}^{3}+C \omega_{2}^{4}+a_{i} \omega^{i}=0, \quad i=1,2,3,4 .
$$

Канонизируя, получаем $\omega_{1}^{4}=\omega_{2}^{3}$ (если $B \neq 0$ ) $(7)$. Будем рассматривать общий случай $B \neq 0$.

ЛЕмма Картана [3]. Если между $2 r$ линейными формами $f_{i}, \varphi_{i}$ из $n$-мерного кольиа $\Re[u]$ имеет место тождество

$$
\left[f_{1} \varphi_{1}\right]+\left[f_{2} \varphi_{2}\right]+\cdots+\left[f_{r} \varphi_{r}\right]=0
$$

и система форм $f_{i}-$ ранга $r$, то формы $\varphi_{k}$ линейно выражаются через формы $f_{i}$ с симметричной матрицей коэффициентов.

После дифференцирования внешним образом уравнения (7) и применения леммы Картана получим

$$
\begin{array}{rlr}
-\omega_{3}^{4} & =\omega_{2}^{1}+a_{11} \omega_{1}^{3}+a_{12} \omega_{2}^{3}+a_{13} \omega_{2}^{4}+a_{1, i+3} \omega^{i}=0, \\
-\omega_{4}^{4} & =-\omega_{1}^{1}+\omega_{2}^{2}-\omega_{3}^{3}+a_{12} \omega_{1}^{3}+a_{22} \omega_{2}^{3}+a_{23} \omega_{2}^{4}+a_{2, i+3} \omega^{i}=0, & \\
\omega_{4}^{3} & =-\omega_{1}^{2}+a_{13} \omega_{1}^{3}+a_{23} \omega_{2}^{3}+a_{33} \omega_{2}^{4}+a_{3, i+3} \omega^{i}=0, & \\
\omega_{12}^{3} & =\omega_{11}^{4}+\cdots, & \\
\omega_{23}^{3} & =\omega_{13}^{4}+\cdots, \\
\omega_{24}^{3} & =\omega_{14}^{4}+\cdots,
\end{array}
$$

Матрица коэффициентов - симметричная. После канонизации некоторые из коэффициентов $a_{i j}$ можно привести к нулю. Остальные будут образовывать геометрический объект: это будут коэффициенты $a_{11}, a_{12}, a_{22}, a_{23}$ и $a_{33}$. Для изучения геометрического смысла тех геометрических объектов, которые будут получены далее, 
важно рассмотреть другую интерпретацию этого восьмимерного пространства $M^{(8)}$, а именно, рассмотреть четырехмерное пространство $M^{(4)}$. С каждой его точкой $\left(u_{1}\right.$, $\left.u_{2}, u_{3}, u_{4}\right)$ свяжем два вектора

$$
\begin{aligned}
\vec{e}_{1} & =\bar{M}_{u_{1}}+\bar{M}_{u_{3}} \cdot p_{1}+\bar{M}_{u_{4}} \cdot p_{3}, \\
\vec{e}_{2} & =\bar{M}_{u_{2}}+\bar{M}_{u_{3}} \cdot p_{2}+\bar{M}_{u_{4}} \cdot p_{4} .
\end{aligned}
$$

Эти два вектора определяют плоскость. Каждой точке $\left(u_{1}, \ldots, u_{4}, p_{1}, \ldots, p_{4}\right)$ восьмимерного пространства $M^{(8)}$ поставим в соответствие точку $M\left(u_{1}, u_{2}, u_{3}, u_{4}\right)$ и двумерную плоскость. Легко видеть, что для интегрального многообразия первого исходного уравнения

$$
u=u(x, y), \quad v=v(x, y), \quad p_{1}=\frac{\partial u}{\partial x}, \quad p_{2}=\frac{\partial u}{\partial y}, \quad p_{3}=\frac{\partial v}{\partial x}, \quad p_{4}=\frac{\partial v}{\partial y}
$$

эта двумерная плоскость будет касательным элементом.

Тогда первое уравнение

$$
\omega_{1}^{4}=\omega_{2}^{3}
$$

выделит подмногообразие этих двумерных элементов. Зафиксируем в этом четырехмерном пространстве точку, т.е. положим

$$
\omega^{i}=0, \quad i=1,2,3,4 .
$$

Назовем эту точку нулевой точкой пространства. Преобразования нашей группы, которые сохраняют точку, образуют иентроаффинную группу. Рассмотрим структурные уравнения (4) для инвариантных форм нашей задачи. Если $\omega^{i}=0$, то эти уравнения будут такими:

$$
D \omega_{k}^{i}=\left[\omega_{l}^{i} \omega_{k}^{l}\right], \quad i=1,2,3,4 .
$$

Остальных уравнений не будет. Уравнения (9) являются структурными уравнениями центроафбинной группь. С каждой фиксированной точкой $\omega^{i}=0$ будет связано четырехмерное многообразие двумерных плоскостей. Рассмотрим проективную интерпретацию этого образа. В пересечении с трехмерной несобственной гиперплоскостью, не проходящей через нулевую точку пространства, это центроаффинное пространство образует проективное пространство со структурными уравнениями (9) и подвижным репером - тетраэдром $A_{1} A_{2} A_{3} A_{4}$, инфинитезимальные преобразования которого имеют вид

$$
d A_{i}=\omega_{i}^{k} A_{k}, \quad i, k=1,2,3,4 .
$$

Трехмерное многообразие двумерных плоскостей в центроаффинном пространстве, выделяемое уравнением (7), в пересечении с трехмерной несобственной гиперплоскостью, не проходящей через нулевую точку пространства, даст комплекс прямых. С каждой прямой комплекса сопоставим тетраэдр $A_{1} A_{2} A_{3} A_{4}$, ребро которого совпадает с этой прямой. Проводимая канонизация уравнения $(A=0, C=0$, $B=1)$ получает геометрический смысл выбора тетраэдра $A_{1} A_{2} A_{3} A_{4}$, наиболее удобным образом присоединенного к прямой комплекса. А именно, обращение в нуль коэффициента $A$ означает, что в качестве плоскости $A_{2} A_{1} A_{3}$ нашего тетраэдра выбрана плоскость, касательная к конусу лучей комплекса, имеющему вершину в точ- 
ке $A_{2}$; обращение в нуль коэффициента $C$ достигается таким выбором положения координатного тетраэдра, при котором плоскость $A_{1} A_{2} A_{4}$ будет касательной к конусу лучей комплекса, имеющему вершину в точке $A_{1}$. Коэффициент $B$ приводится к 1 нормированием координат вершины $A_{4}$ тетраэдра. Объект $\left(a_{11}, a_{12}, a_{22}, a_{23}, a_{33}\right)$ характеризует этот комплекс.

Тем самым классификация в точке уравнения первого порядка нашей задачи сводится к классификации комплексов. Эта классификация известна, а следовательно, известен и геометрический смысл первого уравнения. Например, если $a_{11}=a_{12}=$ $a_{22}=a_{23}=a_{33}=0$, то соответствующий комплекс - линейный. Для этого случая рассмотрим систему уравнений

$$
\begin{gathered}
\omega_{1}^{4}=\omega_{2}^{3} \\
\bar{A} \omega_{11}^{3}+\bar{B} \omega_{22}^{3}+\bar{C} \omega_{11}^{4}+\bar{D} \omega_{22}^{4}+\alpha \omega_{1}^{3}+\beta \omega_{2}^{3}+\gamma \omega_{2}^{4}+e_{i} \omega^{i}=0, \quad i=1,2,3,4 .
\end{gathered}
$$

Второе уравнение этой системы можно разрешать относительно той или другой трехиндексной формы (в общем случае), а затем по-разному канонизировать полученное при этом уравнение. Геометрический смысл такой канонизации выясняется при рассмотрении новой интерпретации пространства представления группы, относительно преобразований которой изучаются уравнения задачи. При этой новой интерпретации образующим элементом является точка, плоский элемент и проходящий через него элемент второго порядка. С каждой точкой в пространстве и плоским элементом связано трехмерное многообразие элементов второго порядка. Действительно, на шесть производных второго порядка имеем три уравнения: два от продолжения первого уравнения и второе - данное уравнение. Введем понятие характеристики. Характеристика - это такой линейный элемент, через который проходит большее число двумерных интегральных элементов, чем через соседние элементы. Вдоль характеристических направлений нельзя задавать начальные условия для системы.

Если считать, что $\bar{C} \neq 0$ и разрешать второе уравнение системы (11) относительно $\omega_{11}^{4}$, то будет выделен некоторый специальный случай данной в самом начале системы дифференциальных уравнений. Действительно, дальнейшая канонизация приведет лишь к таким возможным случаям:

1) $\omega_{11}^{4}=\omega_{22}^{3}$ - три различные действительные характеристики;

2) $\omega_{11}^{4}=0$ - две совпавшие характеристики и еще одна.

Если считать, что $\bar{B} \neq 0$ и разрешать второе уравнение относительно $\omega_{22}^{3}$, то мы также получим некоторый специальный случай, так как в результате канонизации возникнет такое новое уравнение:

1) $\omega_{22}^{3}=0$ - две совпавшие характеристики и еще одна.

Если $\bar{A} \neq 0$, то можно разрешить второе уравнение системы (11) относительно $\omega_{11}^{3}$. Канонизация в этом случае показывает, что мы имеем систему общего типа:

1) $\omega_{11}^{3}=\omega_{22}^{3}$ - три различные действительные характеристики;

2) $\omega_{11}^{3}=\omega_{11}^{4}-$ две совпавшие характеристики и еще одна;

3) $\omega_{11}^{3}=0-$ три совпавшие характеристики.

Такой же общий тип системы будет, если $\bar{D} \neq 0$ и второе уравнение системы (11) разрешено относительно $\omega_{22}^{4}$. Будем дальше рассматривать именно этот случай. После канонизации такой системы второе уравнение может быть приведено к таким уравнениям:

1) $\omega_{22}^{4}=\omega_{11}^{4}$ - три различные действительные характеристики; 
2) $\omega_{22}^{4}=\omega_{11}^{3}$ - три различные характеристики (из них две комплексно-сопряженные);

3) $\omega_{22}^{4}=\omega_{22}^{3}-$ две совпавшие и одна;

4) $\omega_{22}^{4}=0$ - три совпавшие характеристики.

Тем самым, получается первоначальная классификация этой системы. Возьмем первую систему (самого общего вида, когда у нее три характеристики действительные, не совпадающие друг с другом):

$$
\begin{gathered}
\omega_{1}^{4}=\omega_{2}^{3}, \\
\omega_{22}^{4}=\omega_{11}^{4} .
\end{gathered}
$$

Дальнейшее продолжение и возможная канонизация приводит к таким уравнениям при условии линейного комплекса $\left(a_{11}=a_{12}=a_{22}=a_{23}=0\right)$ :

$$
\begin{gathered}
\omega_{2}^{1}=b_{11} \omega_{11}^{3}+b_{12} \omega_{11}^{4}+b_{13} \omega_{22}^{3}+a \omega_{1}^{3}+b \omega_{2}^{3}+e \omega_{2}^{4}+b_{1, i+6} \omega^{i} \\
\omega_{1}^{2}=b_{12} \omega_{11}^{3}+b_{22} \omega_{11}^{4}+b_{23} \omega_{22}^{3}-e \omega_{1}^{3}+g \omega_{2}^{3}+f \omega_{2}^{4}+b_{2, i+6} \omega^{i} \\
\omega_{1}^{1}-\omega_{2}^{2}=b_{13} \omega_{11}^{3}+b_{23} \omega_{11}^{4}+b_{33} \omega_{22}^{3}-b \omega_{1}^{3}+c \omega_{2}^{3}+g \omega_{2}^{4}+b_{3, i+6} \omega^{i} \\
-2 \omega_{13}^{4}=\ldots \\
2 \omega_{23}^{4}-\omega_{22}^{1}-2 \omega_{14}^{4}+\omega_{11}^{1}=\ldots \\
2 \omega_{24}^{4}-\omega_{22}^{2}+\omega_{11}^{2}=\ldots \\
\omega_{22 i}^{4}-\omega_{11 i}^{4}=0 .
\end{gathered}
$$

Коэффициенты при трехиндексных формах образуют геометрический объект, а коэффициенты при двухиндексных формах являются относительными инвариантами нашей группы (при условии фиксации точки $\omega^{i}=0, i=1,2,3,4$. Эти объекты позволяют классифицировать эту систему более подробно. А именно, если зафиксировать точку, плоский элемент и двумерный элемент, то три характеристики в пересечении с прямой комплекса определят тройку точек. Всегда можно считать, что это точки $A_{1}, A_{2}, A_{1}+A_{2}$. Если фиксировать только исходную точку и плоский элемент, то таких троек будет трехпараметрическое семейство. Таким образом, свойства системы в точке сводятся к свойствам такой геометрической конфигурации: комплекс прямых и на каждой прямой трехпараметрическое многообразие троек точек. Если все $a_{11}=a_{12}=a_{22}=a_{23}=a_{33}=0, b_{i j}=0, i=1,2,3,4$, все $a=b=c=-e=-f=g=0$ (объект равен нулю), и все относительные инварианты равны нулю, то на каждом луче эти тройки неподвижны и в трехмерной несобственной гиперплоскости существует неподвижная прямая, через которую проходят три фиксированные плоскости, которые на каждой прямой комплекса высекают эту тройку точек. Значит, можно, во-первых, производить классификацию по уменьшению произвола этих троек на каждом луче комплекса, т.е. по снижению ранга матрицы

$$
\left(\begin{array}{ccc}
a & b & e \\
-e & g & f \\
-b & c & g
\end{array}\right),
$$

а во-вторых, по существованию на прямой $A_{1} A_{2}$ в комплексе таких точек $A_{1}+A_{2}$, которые описывали бы при движении прямой не все пространство, а двумерную 
поверхность. Задача приводит к уравнению 4-го порядка

$$
a h^{4}-2 b h^{3}+\left(2 e A_{1}+A_{2} c\right) h^{2}+2 g h-f=0
$$

и, значит, вообще говоря, возможна такая классификация:

1) на прямой вообще нет таких точек;

2) 4 различные точки;

3) различные случаи совпадения таких точек;

4) случай тождественного выполнения такого уравнения.

Поскольку нами рассматривается такая система дифференциальных уравнений, для которой все три характеристики действительные и различные, для уравнения (17) остаются лишь такие возможности:

1) нет ни одного корня (т.е. ни одна из точек прямой $A A_{2}$ комплекса не описывает поверхность при движении луча);

2) существуют четыре различных корня, т.е. 4 точки $A_{1}, A_{2}, A_{1}+A_{2}$ и $A$, описывающие поверхности (все различные);

3) существует один двойной корень и два различных.

В “двойной” точке может быть только совпадение точки $A$ с какой-либо из точек $A_{1}$, $A_{2}$ или $A_{1}+A_{2}$ (точки $A_{1}, A_{2}, A_{1}+A_{2}$ по предположению не могут совпадать между собой). Других случаев не будет. Условия, при которых точки $A_{1}\left(h_{1}=0\right), A_{2}$ $\left(h_{2}=\infty\right), A_{1}+A_{2}\left(h_{3}=1\right)$ описывают поверхности при движении прямой комплекса, таковы:

$$
f=0, \quad a-2 b+2 e+c+2 g-f=0
$$

(соответственно).

Если эти условия выполняются одновременно, то координаты четвертой точки $A$, описывающей поверхность, определяются по формуле

$$
h_{4}=\frac{-g}{b}=-\lambda, \quad \frac{g}{b}=\lambda=\text { const. }
$$

Дифференциальные уравнения нашей системы в точке, т.е. при $\omega^{i}=0, i=1,2,3,4$, при этом станут такими уравнениями Пфаффа:

$$
\begin{aligned}
& \omega_{1}^{4}=\omega_{2}^{3}, \\
& \omega_{4}^{1}=\omega_{3}^{2} \text {, } \\
& \omega_{2}^{1}=b \omega_{2}^{3}+e \omega_{2}^{4}, \\
& \omega_{1}^{2}=-e \omega_{1}^{3}+\lambda b \omega_{2}^{3}, \\
& \omega_{1}^{1}-\omega_{2}^{2}=-b \omega_{1}^{3}+2(e+(\lambda-1)) b \omega_{2}^{3}+\lambda b \omega_{2}^{4} \\
& \omega_{3}^{4}=-\omega_{2}^{1}, \\
& \omega_{4}^{3}=-\omega_{1}^{2}, \\
& \omega_{4}^{4}-\omega_{3}^{3}=\omega_{1}^{1}-\omega_{2}^{2} .
\end{aligned}
$$

Классификация этой системы может быть более детально произведена по снижению ранга матрицы коэффициентов, стоящих в правых частях уравнений (13):

$$
D=\operatorname{det}\left(\begin{array}{ccc}
0 & b & e \\
-e & b & 0 \\
-b & 2(e+(-1) b) & b
\end{array}\right) .
$$


Этот определитель матрицы, в общем случае, не равен нулю тождественно, значит, ранг этой матрицы равен трем. Произвол троек точек на лучах комплекса не снизится, если на каждом луче комплекса найдутся четыре точки, описывающие поверхности при смещении этого луча. Ранг этой матрицы уменьшится до двух, если либо $e=0$, либо $b=-e$, либо $\lambda b+e=0$.

Таким образом, при выполнении одного из условий (18) точки $A_{1}, A_{2}, A_{1}+A_{2}, A$ прямой комплекса будут описывать поверхности, и произвол троек точек пересечения характеристик с прямой комплекса уменьшится до двух:

$$
e=0, \quad b=-e \quad \text { либо } \quad \lambda b+e=0 .
$$

В работе [7] рассматривался случай с тремя различными характеристиками на каждом интегральном многообразии. В проективном пространстве, ассоциированном с касательным пространством к $M^{(4)}$ в произвольной точке, такая система задает комплекс прямых. На каждой прямой из прямых комплекса имеется семейство троек точек, зависящее, вообще говоря, от трех параметров, а в частных случаях от меньшего числа параметров. Была проведена классификация таких геометрических образов. В частности, выяснилось, что самым простым будет случай, когда комплекс линейный, и на каждой его прямой три характеристические точки определяются единственным образом как пересечение этой прямой с тремя постоянными плоскостями, проходящими через одну прямую, принадлежащую комплексу. В этом случае вектор реперов, присоединенных к системе в точке, удовлетворяет дифференциальным уравнениям

$$
\begin{aligned}
& d A_{1}=\widetilde{\omega} A_{1}+\widetilde{\omega}_{1}^{3} A_{3}+\widetilde{\Omega} A_{4}, \\
& d A_{3}=\widetilde{\Theta} A_{3}, \\
& d A_{2}=\widetilde{\omega} A_{2}+\widetilde{\Omega} A_{3}+\widetilde{\omega}_{2}^{4} A_{4}, \\
& d A_{4}=\widetilde{\Theta} A_{4}
\end{aligned}
$$

(векторы $A_{1}, A_{2}$ принадлежат площадке; ее характеристические направления определяются векторами $A_{1}, A_{2}, A_{1}-A_{2}$; прямая пересечения трех плоскостей определяется векторами $\left.A_{3}, A_{4}\right)$. При этом будут выполняться дифференциальные уравнения

$$
\begin{aligned}
d \omega^{1} & =\left[\omega \omega^{1}\right]+\cdots, \\
d \omega^{2} & =\left[\omega \omega^{2}\right]+\cdots, \\
d \omega^{3} & =\left[\omega_{1}^{3} \omega^{1}\right]+\left[\Omega \omega^{2}\right]+\left[\Theta \omega^{3}\right]+\cdots, \\
d \omega^{4} & =\left[\Omega \omega^{1}\right]+\left[\omega_{2}^{4} \omega^{2}\right]+\left[\Theta \omega^{4}\right]+\cdots,
\end{aligned}
$$

где формы, входящие в (24), получаются из соответствующих форм без значков в (25) при фиксации точки в $M^{(4)}$. Не выписанные члены являются линейными комбинациями форм $\omega^{i}$. В данном случае изучение системы совпадает с изучением $g$-структуры в $M^{(4)}$. Группа $g$ оставляет инвариантным в несобственном проективном пространстве линейный комплекс и три указанные выше плоскости, а семейство реперов определяется уравнениями (24).

Мы рассмотрим еще более специальный случай, когда не выписанные члены в (25) приводятся к нулю, т.е. первая структурная функция данной $g$-структуры равна 
нулю:

$$
\begin{aligned}
d \omega^{1} & =\left[\omega \omega^{1}\right], \\
d \omega^{2} & =\left[\omega \omega^{2}\right], \\
d \omega^{3} & =\left[\omega_{1}^{3} \omega^{1}\right]+\left[\Omega \omega^{2}\right]+\left[\Theta \omega^{3}\right], \\
d \omega^{4} & =\left[\Omega \omega^{1}\right]+\left[\omega_{2}^{4} \omega^{2}\right]+\left[\Theta \omega^{4}\right] .
\end{aligned}
$$

Сравнение (26) с (4) дает

$$
\widetilde{\omega}=\widetilde{\omega}_{1}^{1}=\widetilde{\omega}_{2}^{2}, \quad \widetilde{\Omega}=\widetilde{\omega}_{3}^{3}=\widetilde{\omega}_{4}^{4},
$$

остальные формы $\widetilde{\omega}_{i}^{k}$ равны нулю. Дифференцируя уравнение $(26)$, получим

$$
\begin{aligned}
{\left[\Delta \omega_{1}^{3} \omega^{1}\right]+\left[\Delta \Omega \omega^{2}\right]+\left[d \Theta \omega^{3}\right] } & =0 \\
{\left[\Delta \Omega \omega^{1}\right]+\left[\Delta \omega_{2}^{4} \omega^{2}\right]+\left[d \Theta \omega^{4}\right] } & =0 \\
{\left[d \omega \omega^{1}\right] } & =0 \\
{\left[d \omega \omega^{2}\right] } & =0,
\end{aligned}
$$

где

$$
\begin{aligned}
\Delta & =d \omega_{1}^{3}-\left[\omega_{1}^{3} \omega\right]-\left[\Theta \omega_{1}^{3}\right], \\
\Delta \Omega & =d \Omega-[\Omega \omega]-[\Theta \Omega], \\
\omega_{2}^{4} & =d \omega_{2}^{4}-\left[\omega_{2}^{4} \omega\right]-\left[\Theta \omega_{2}^{4}\right] .
\end{aligned}
$$

После применения к уравнениям (28) обобщенной, а затем простой леммы Картана, а также учитывая, что уравнения системы налагают на формы три уравнения, а характеристические направления определяются уравнениями $\omega^{1}=0, \omega^{2}=0$, $\omega^{1}+\omega^{2}=0$, вводя обозначения $\omega_{12}^{3}=\omega_{11}^{4}=\omega_{22}^{3}=\omega_{12}^{4}=\Omega_{0}$, получим

$$
\begin{aligned}
\Delta \omega_{1}^{3} & =\left[\omega_{11}^{3} \omega^{1}\right]+\left[\Omega_{0} \omega^{2}\right]+A_{1, i k}^{3}\left[\omega^{i} \omega^{k}\right], \\
\Delta \omega_{2}^{4} & =\left[\Omega_{0} \omega^{1}\right]+\left[\omega_{22}^{4} \omega^{2}\right]+A_{2, i k}^{4}\left[\omega^{i} \omega^{k}\right], \\
\Delta \Omega & =\left[\Omega_{0}\left(\omega^{1}+\omega^{2}\right)\right]+B_{i k}\left[\omega^{i} \omega^{k}\right], \\
\Delta \Theta & =A_{i k}\left[\omega^{i} \omega^{k}\right] .
\end{aligned}
$$

Вводя преобразования форм

$$
\omega_{11}^{3} \rightarrow \omega_{11}^{3}+u_{i}^{3} \omega^{k}, \quad \omega_{22}^{4} \rightarrow \omega_{22}^{4}+u_{i}^{4} \omega^{k}, \quad \Omega_{0} \rightarrow \Omega_{0}+v^{i} \omega^{i},
$$

не меняющие уравнений (30), можно преобразовать их к такому виду

$$
\begin{aligned}
\Delta \omega_{1}^{3} & =\left[\omega_{11}^{3} \omega^{1}\right]+\left[\Omega_{0} \omega^{2}\right]-A_{12}\left[\omega^{2} \omega^{3}\right]+B_{14}\left[\omega^{2} \omega^{4}\right]+A_{14}\left[\omega^{3} \omega^{4}\right], \\
\Delta \omega_{2}^{4} & =\left[\Omega_{0} \omega^{1}\right]+\left[\omega_{22}^{4} \omega^{2}\right]+B_{23}\left[\omega^{1} \omega^{3}\right]+A_{12}\left[\omega^{1} \omega^{4}\right]-A_{23}\left[\omega^{3} \omega^{4}\right], \\
\Delta \Omega & =\left[\Omega_{0}\left(\omega^{1}+\omega^{2}\right)\right]+B_{23}\left[\omega^{2} \omega^{3}\right]+B_{14}\left[\omega^{1} \omega^{4}\right]+A\left[\omega^{3} \omega^{4}\right], \\
d \Theta & =A_{12}\left[\omega^{1} \omega^{2}\right]+A\left[\omega^{1} \omega^{3}\right]+A_{14}\left[\omega^{1} \omega^{4}\right]+A_{23}\left[\omega^{2} \omega^{3}\right]+A\left[\omega^{2} \omega^{4}\right]+A_{34}\left[\omega^{3} \omega^{4}\right], \\
d \omega & =K\left[\omega^{1} \omega^{2}\right] .
\end{aligned}
$$

Заметим, что изучаемая нами система эквивалентна системе внешних дифференциальных уравнений

$$
\omega^{3}=0, \quad \omega^{4}=0, \quad\left[\left(\omega_{1}^{3}-\Omega\right) \omega^{1}\right]=0, \quad\left[\left(\omega_{2}^{4}-\Omega\right) \omega^{2}\right]=0, \quad\left[\Omega\left(\omega^{1}+\omega^{2}\right)\right]=0
$$


на многообразии $M^{(7)}$. Дифференцируя уравнения (31) внешним образом и приводя подобные члены, получим

$$
\begin{aligned}
{\left[\Delta \omega_{11}^{3} \omega^{1}\right]+\left[\Delta \Omega_{0} \omega^{2}\right]-\left[\Delta A_{12} \omega^{2} \omega^{3}\right]+\left[\Delta B_{14} \omega^{2} \omega^{4}\right]+\left[\Delta A_{14} \omega^{3} \omega^{4}\right] } & =0 \\
{\left[\Delta \Omega_{0} \omega^{1}\right]+\left[\Delta \omega_{22}^{4} \omega^{2}\right]+\left[\Delta B_{23} \omega^{1} \omega^{3}\right]+\left[\Delta A_{12} \omega^{1} \omega^{4}\right]-\left[\Delta A_{23} \omega^{3} \omega^{4}\right] } & =0 \\
{\left[\Delta \Omega_{0}\left(\omega^{1}+\omega^{2}\right)\right]+\left[\Delta B_{23} \omega^{2} \omega^{3}\right]+\left[\Delta B_{14} \omega^{1} \omega^{4}\right]+\left[\Delta A \omega^{3} \omega^{4}\right] } & =0 \\
{\left[\Delta A_{12} \omega^{1} \omega^{2}\right]+\left[\Delta A\left(\omega^{2} \omega^{4}-\omega^{1} \omega^{3}\right)\right]+\left[\Delta A_{14} \omega^{1} \omega^{4}\right] } & \\
+\left[\Delta A_{23} \omega^{2} \omega^{3}\right]+\left[\Delta A_{34} \omega^{3} \omega^{4}\right] & =0 \\
{\left[\Delta K \omega^{1} \omega^{2}\right] } & =0
\end{aligned}
$$

где введены такие обозначения:

$$
\begin{aligned}
& \Delta K= d K+2 K \omega \\
& \Delta A_{34}=d A_{34}+2 A_{34} \Theta \\
& \Delta A=d A+A \omega+A \Theta+A_{34} \Omega \\
& \Delta A_{14}=d A_{14}+A_{14} \omega+A_{14} \Theta+A_{34} \omega_{1}^{3} \\
& \Delta A_{23}=d A_{23}+A_{23} \omega+A_{23} \Theta-A_{34} \omega_{2}^{4} \\
& \Delta A_{12}=d A_{12}+2 A_{12} \omega+A_{14} \omega_{2}^{4}-A_{23} \omega_{1}^{3} \\
& \Delta B_{14}= d B_{14}+2 B_{14} \omega+A \omega_{1}^{3}+A_{14} \Omega-2 A \Omega \\
& \Delta B_{23}= d B_{23}+2 B_{23} \omega-A \omega_{2}^{4}+A_{23} \Omega+2 A \Omega, \\
& \Delta \Omega_{0}=d \Omega_{0}-2\left[\Omega_{0} \omega\right]+\left[\Omega_{0} \Theta\right]-\frac{1}{2} K\left[\Omega\left(\omega^{1}-\omega^{2}\right)\right]+\frac{1}{2} A_{12}\left[\Omega\left(\omega^{1}-\omega^{2}\right)\right] \\
& \quad-\frac{1}{2} B_{23}\left[\omega_{1}^{3}\left(\omega^{1}-\omega^{2}\right)\right]+\frac{1}{2} B_{14}\left[\omega_{2}^{4}\left(\omega^{1}-\omega^{2}\right)\right]-2 A\left[\Omega \omega^{4}\right]+2 A\left[\Omega \omega^{3}\right], \\
& \Delta \omega_{11}^{3}=d \omega_{11}^{3}-2\left[\omega_{11}^{3} \omega\right]+\left[\omega_{11}^{3} \Theta\right]+K\left[\omega_{1}^{3} \omega^{2}\right]-\frac{1}{2} K\left[\Omega \omega^{2}\right] \\
& \quad-2 A_{12}\left[\omega_{1}^{3} \omega^{2}\right]+\frac{1}{2} A_{12}\left[\Omega \omega^{2}\right]+B_{14}\left[\Omega \omega^{2}\right]+\frac{1}{2} B_{14}\left[\omega_{2}^{4} \omega^{2}\right] \\
& \quad-\frac{1}{2} B_{23}\left[\omega_{1}^{3} \omega^{2}\right]-2 A_{14}\left[\omega_{1}^{3} \omega^{4}\right]+A_{14}\left[\Omega \omega^{3}\right]+A\left[\omega_{1}^{3} \omega^{3}\right], \\
& \quad+\frac{1}{2} B_{23}\left[\omega_{1}^{3} \omega^{1}\right]-2 A_{23}\left[\omega_{2}^{4} \omega^{3}\right]+A_{23}\left[\Omega \omega^{4}\right]-A\left[\omega_{2}^{4} \omega^{4}\right] . \\
& \Delta \omega_{22}^{4}=d \omega_{22}^{4}-2\left[\omega_{22}^{4} \omega\right]+\left[\omega_{22}^{4} \Theta\right]-K\left[\omega_{2}^{4} \omega^{1}\right]+\frac{1}{2} K\left[\Omega \omega^{1}\right] \\
& \quad+2 A_{12}\left[\omega_{2}^{4} \omega^{1}\right]-\frac{1}{2} A_{12}\left[\Omega \omega^{1}\right]+B_{23}\left[\Omega \omega^{1}\right]-\frac{1}{2} B_{14}\left[\omega_{2}^{4} \omega^{1}\right]
\end{aligned}
$$

Из этих уравнений видно, что $K$ и $A_{34}$ являются относительными инвариантами, а ряд других коэффициентов образуют линейные геометрические объекть:

$$
\begin{aligned}
& \left(A_{34}, \lambda A+\mu A_{14}+\nu A_{23}\right), \quad \lambda=\text { const, } \quad \mu=\text { const, } \quad \nu=\text { const }, \\
& \left(A_{34}, A_{14}, A, B_{14}\right), \quad\left(A_{34}, A, A_{23}, B_{23}\right), \quad\left(A_{34}, A_{14}, A_{23}, A_{12}\right)
\end{aligned}
$$

и другие.

Все коэффициенты вместе также образуют линейный геометрический объект (структурную функцию второго порядка g-структуры). Выясним геометрический 
смысл обращения в нуль этих объектов. Вполне интегрируемая система $\omega^{1}=0$, $\omega^{2}=0$ определяет расслоение многообразия $M^{(4)}$ с базисным многообразием $M^{(2)}$, которое мы будем называть пространством независимых переменных.

Каждое из уравнений $\omega^{1}=0, \omega^{2}=0, \omega^{1}+\omega^{2}=0$ вполне интегрируемо. Они определяют на $M^{(2)}$ три семейства кривых. $K$ является кривизной этой три-ткани в смысле Бляшке [8]. Аналогично, на каждом слое расслоения $M^{(4)}$, т.е. на интегральном многообразии системы $\omega^{1}=0, \omega^{2}=0$ уравнения $\omega^{3}=0, \omega^{4}=0$ определяют три-ткань, кривизна которой равна $A_{34}$.

Обращение в нуль объекта $\left(A_{34}, \lambda A+\mu A_{14}+\nu A_{23}\right)$ необходимо и достаточно для полной интегрируемости системы Пфаффа $\omega^{1}=0, \omega^{2}=0, \lambda \Omega+\mu \omega_{1}^{3}+\nu \omega_{2}^{4}=0$ в многообразии $M^{(7)}$. Аналогично, обращение в нуль объекта $\left(A_{34}, A, A_{14}, A_{23}, B_{23}+A_{12}\right)$ необходимо и достаточно для полной интегрируемости системы $\omega^{1}=0, \Omega-\omega_{1}^{3}=0$. В этом случае уравнение $\left[\left(\omega_{1}^{3}-\Omega\right) \omega^{1}\right]=0$ является самостоятельным дифберенциальным уравнением в системе (32). Подставляя его решения в остальные уравнения системы, приходим к более простой системе дифференциальных уравнений, т.е. система допускает промежуточный интеграл. Аналогичную роль для уравнений $\left[\left(\omega_{2}^{4}-\Omega\right) \omega^{2}\right]=0$ играет обращение в нуль объекта

$$
\left(A_{34}, A, A_{14}, A_{23}, B_{23}, B_{14}-A_{12}\right),
$$

а для уравнения $\left[\Omega\left(\omega^{1}+\omega^{2}\right)\right]=0-$ объекта

$$
\left(A_{34}, A, A_{14}, A_{23}, B_{23}, B_{14}\right) \text {. }
$$

В частности, получаем следующий результат.

Теорема. Если у системы изучаемого вида две из систем Пфаффа

$$
\begin{aligned}
& \omega_{1}^{3}-\Omega=0, \quad \omega_{2}^{4}-\Omega=0, \quad \Omega=0, \\
& \omega^{1}=0, \quad \omega^{2}=0, \quad \omega^{1}+\omega^{2}=0,
\end{aligned}
$$

вполне интегрируемы, то это верно и для третъей системъ.

Такие уравнения возникают при решении различных прикладных задач гидродинамики, физики атмосферы, физики плазмы. Здесь следует сослаться на системы уравнений Соболева, а также его учеников. Системы уравнений указанного типа применяются и для описания реальных физико-химических процессов. Эти результаты изложены в книге Акрамова [9]. Очень интересные примеры таких систем удалось найти на страницах книги [10].

Автор выражает глубокую благодарность за помощь в работе над статьей всему коллективу кафедры математического анализа механико-математического факультета МГУ, особенно профессору Т. П. Лукашенко, профессору Л. Е. Евтушику, доценту Т. В. Родионову.

Автор выражает также глубокую благодарность А. Г. Кушнеру за предоставление возможности познакомиться с книгой [10].

\section{СПИСОК ЦИТИРОВАННОЙ ЛИТЕРАТУРЫ}

[1] Э. Картан, Теория конечнъх непреръвных групп и дифференииалъная геометрия, изложенные методом подвижного репера, Изд-во МГУ, М., 1963. 
[2] Э. Картан, Избранные труды, МЦНМО, М., 1998.

[3] С. П. Фиников, Метод внешних форм Картана в дифференииальной геометрии. Теория совместимости систем дифференциальных уравнений в полных дифференциалах и в частных производных, Гостехиздат, М.-Л., 1948.

[4] Г. Ф. Лаптев, “Дифференциальная геометрия погруженных многообразий. Теоретико-групповой метод дифференциально-геометрических исследований”, Tp. MMO, 2 (1953), 275-382.

[5] А. М. Васильев, "Системы трех дифференциальных уравнений с частными производными первого порядка при трех неизвестных функциях и двух независимых переменных (локальная теория)", Матем. сб., 70:4 (1966), 457-480.

[6] А.М. Васильев, Теория дифференииально-геометрических структур, Изд-во МГУ, M., 1987.

[7] Л.Н. Орлова, "Система двух дифференциальных уравнений с частными производными первого и второго порядка при двух неизвестных функциях и двух независимых переменных", Вопросы дифференииальной и неевклидовой геометрии, Ученые записки, № 271, Моск. гос. пед. ин-т им. В. И. Ленина, М., 1967, 103-112.

[8] В. Бляшке, Введение в геометрию тканей, Физматгиз, М., 1959.

[9] Т.А. Акрамов, Дифференциальные уравнения и их приложения в моделировании физико-химических прочессов, Баширский гос. ун-т., Уфа, 2000.

[10] A. Kushner, V. Lychagin, V. Rubtsov, Contact Geometry and Non-Linear Differential Equations, Encyclopedia Math. Appl., 101, Cambridge Univ. Press, Cambridge, 2007.

\section{Л.Н. Орлова}

Московский государственный строительный университет E-mail: Meek@mail.ru
Поступило 02.07 .2007

Исправленный вариант 09.06.2008 